\title{
PET and PET/CT in gastrointestinal stromal tumours: the unanswered questions and the potential newer applications
}

\author{
Sandip Basu
}

Received: 24 August 2009/Accepted: 3 February 2010/Published online: 18 March 2010

(C) Springer-Verlag 2010

The scientific evidence over the last decade has demonstrated the revolutionary impact of FDG PET as an excellent molecular imaging tool for evaluating early therapeutic response of gastrointestinal stromal tumours (GIST) to imatinib mesylate [1]. By now, it is quite evident that the early response of GIST to imatinib therapy cannot be reliably monitored by the World Health Organization (WHO) criteria, Response Evaluation Criteria in Solid Tumors (RECIST) or the Southwest Oncology Group (SWOG) criteria for assessing treatment response that are solely based upon changes in the tumour size measured by morphological imaging modalities. The results have now been well validated in multiple head-to-head prospective studies with sufficient statistical strength [2-13] that have convincingly proven that FDG PET or PET/CT imaging has been the imaging modality of choice to assess metabolic activity in this group of tumours. Comparing PET activity before and after the start of tyrosine kinase inhibitors provides critical information in identifying active disease and for the assessment of the response to drug treatment. While this has been the major thrust and the frequently highlighted aspect of FDG PET imaging in the management of GISTs, there are other practical issues where this powerful imaging modality can be of great help, and answering these clinical concerns is likely to further enhance the prospects of PET-guided personalized medicine in GIST.

Tailoring and selecting the optimal treatment dose is of great benefit in minimizing the toxic effects of this therapy.

\footnotetext{
S. Basu $(\bowtie)$

Radiation Medicine Centre (BARC),

Tata Memorial Hospital Annexe, Parel,

Bombay 400012, India

e-mail: drsanb@yahoo.com
}

The relatively common dose-limiting adverse effects of imatinib as observed in the phase I studies [14] include oedema, nausea, vomiting, rash and musculoskeletal pain. Though generally well tolerated they are particularly common and are of concern for patients who are of smaller size with cachexia, poor performance status and a large disease burden. Many of them quickly develop oedema or a fluid compartmentalization during imatinib therapy. Hence this has a geographic relevance and is of more concern in developing countries than in their Western counterparts. Presently, a starting dose of $400 \mathrm{mg}$ daily is considered appropriate for most patients with GIST, but patients with an exon 9 mutation of the KIT gene usually require a higher dose. A dose increase is considered by the oncologists when they fail to respond. The results of two large phase III trials (EORTC/ISG/AGITG 62005 and SWOG/CALGB/NCI-C/ ECOG/ICAS S0033) have demonstrated similar responses for imatinib doses of $400 \mathrm{mg} /$ day versus $800 \mathrm{mg} /$ day when mutation status is not considered [15]. However, in both trials, dose reductions due to side effects were much more common in the 800 -mg groups than in the $400-\mathrm{mg}$ groups [15]. Severe congestive cardiac failure is another uncommon but recognized side effect of imatinib and it is predicted that cardiotoxicity could be facilitated by the presence of relevant comorbidities such as pre-existing cardiovascular disease or renal failure [16]. The incidence, natural history and risk factors for developing cardiotoxicity is not clearly defined in the clinical context [17]. Preclinical studies indicate that imatinib and sunitinib may be directly toxic to cardiac myocytes and it is described to be more common and severe with sunitinib [17]. All these imply that starting of therapy at lower doses of these drugs may be considered in appropriate cases with a goal of escalating the dose further when required. Molecular imaging with FDG PET by its ability to document early response can play a pivotal role to tailor and 
optimize the dose of imatinib or sunitinib and should be employed more frequently to define the most appropriate dose in an individual. In our experience, low-dose imatinib (200 mg daily) administration in case of documented side effects with the standard $400 \mathrm{mg}$ dose can be quite effective in disease suppression as observed by metabolic FDG PET imaging and should be considered in such settings.

The clinical and pathological criteria to differentiate benign from malignant GIST are not well established and are at times notoriously difficult [18]. Tumour size and mitotic activity are commonly considered major discriminants predictive of biological behaviour. Tumours with size $>5 \mathrm{~cm}$ and mitotic activity counts $>5 / 50$ high power field (HPF) are considered to be malignant and those with size less than $5 \mathrm{~cm}$ and mitotic activity counts $<5 / 50 \mathrm{HPF}$ are likely to be benign. This, however, leaves a grey area in the separation of benign and malignant tumours and the tumour biology depends on several other factors [20]. Comparative studies of KITmutant, PDGFRA-mutant and wild-type GISTs indicate that there are important differences between these groups of tumours and hence a molecular-based classification of GIST appears more appropriate rather than the aforementioned two factors alone [19]. Furthermore, the significant amount of necrosis that is commonly encountered in these tumours can lead to sampling error during anatomical imaging-guided biopsy and yield falsely low mitotic count in the biopsied specimen. We have observed a similar phenomenon in our prospective series [1]. It has been observed that among mitotically inactive tumours there is a small percentage that later metastasize, illustrating that a low mitotic count does not rule out the possibility of malignant behaviour [20]. FDG PET by its ability to measure metabolism can play an important role in characterizing the tumour phenotype by distinguishing metabolically active malignant GISTs from their benign counterparts and also guiding the appropriate site of biopsy. Kamiyama et al. [21] investigated ten patients to explore the usefulness of FDG PET in predicting the malignant potential, where FDG uptake and malignant potential (Ki-67 index and mitotic index) of gastric GISTs had a significant correlation. Similar observations have been made by Yamada et al. [22] in 21 patients and the results of both these studies provide impetus for yet another promising clinical application of this molecular imaging technique in characterizing and grading these tumours. It will be also worthwhile to study whether any difference in FDG uptake pattern exists amongst the various molecular subtypes with differing mutations.

The "metabolic switch-on" in FDG PET imaging in a compliant patient can indicate secondary resistance to imatinib related to newly acquired second mutations, and the role of this imaging modality in the follow-up of the patients who had shown an initial response to this therapy has been recently underscored by various authors $[1,23]$.
The secondary drug resistance can present in the form of an otherwise responding tumour that starts to grow or as appearance of new metastases. It is presumable that metabolic imaging with FDG PET will be able to detect secondary drug resistance ahead of other anatomical imaging modalities. As more drug options become available, the use of FDG PET imaging for this purpose will hopefully become more common in order to select the best drugs at the earliest opportunity in such settings. We have observed similar FDG PET imaging features in disease relapse in patients who were non-compliant and discontinued treatment themselves due to financial constraints [1], which can be encountered in patients of poor socio-economic status. This is related to the cytostatic nature of the therapy with the small molecule tyrosine kinase inhibitors such as imatinib and sunitinib.

Another important observation of recent times has been the rebound of tumour metabolic activity within a few days after stopping imatinib treatment in imatinibresistant GIST patients, who were being contemplated to be started on multitargeted receptor tyrosine kinase inhibitor sunitinib malate [24]. This observation suggests that a fraction of the tumour cell population continues to be responsive to imatinib in these patients, whereas the secondary resistance and disease progression occur in the resistant clone due to a mutation that is resistant to imatinib [23, 24]. The finding can be related to the fact that substantial intra- and interlesional heterogeneity of resistance mutations and gene amplification exist in patients with clinically progressing GIST [25]. This observation is noteworthy as it provides significant insight into this condition and can thereby provide the basis for therapeutic approaches that would include the cocktails of drugs in treating these patients with secondary resistance to imatinib. Furthermore, it is emphasized that the advantages of the combination regimen include not only the opportunity to maximize initial clinical response, but also the possibility to reduce the incidence of developing secondary resistance, as compared to a monotherapy regimen. Till date, this approach is a relatively unexplored area in GIST research and requires examination in further prospective studies. While certainly an attractive option, the principle of a "cocktail" approach with combinations of drugs is relatively complex and requires defining the correct doses of each drug to minimize the related adverse effects on normal cells. Here again, FDG PET imaging results would prove crucial for optimizing the dose of each drug on a case to case basis and thereby minimizing the likelihood of synergistic side effects related to combination therapy.

An important aspect of GISTs is their association with synchronous or metachronous malignancies, the list of which continues to expand [1]. PET/CT, being a whole-body tomographic technique, can be effectively exploited to this 
end for identification of other malignancies with high sensitivity [1].

Platelet-derived growth factor receptor $\alpha$ (PDGFRA) mutations have also been observed in GISTs and it is suggested that mutational activation of KIT or PDGFRA are mutually exclusive, represent two different alternative oncogenic events and show differing clinical characteristics. In a series of 322 GISTs [19], $80.9 \%$ showed KIT mutations, while $7.1 \%$ showed PDGFRA mutations, and $12 \%$ were wild-type. Recent data indicate that the clinical response of GIST to the tyrosine kinase inhibitors is determined by the specific mutation displayed by their tumours. Limited therapeutic effect has been described in PDGFRA exon 18 mutant tumours affecting the activation loop compared to those with PDGFRA exon 12 mutations at the juxtamembrane domain that usually demonstrates a similar response to GISTs with KIT exon 11 mutations. Recently, the promising role of FDG PET in assessing disease activity has been demonstrated in GISTs that are c-KIT negative but PDGFRA mutation positive [26]. An observation that we have made (unpublished data) is the visualization of bone marrow in a sizeable fraction of untreated GIST patients. Except for one patient (where polycythaemia rubra vera was associated) [27], there was no clear-cut pathological condition that could be related to this observation (bone marrow biopsies carried out in a few patients were negative for any pathological condition). Quite a few of them in this subpopulation had a history of gastrointestinal bleeding as the presenting complaint and had a haemoglobin level that was low or at the lower level of normal limits at the time of FDG PET study. It is not clear at this point whether this bone marrow FDG uptake in untreated GIST patients could be related to the rejuvenating marrow in response to blood loss or whether it is something specific to this group of tumours in this subpopulation of patients with GIST.

Newer therapeutic agents [28, 29] are on the horizons that are being particularly tested in the imatinib-resistant tumours. These include heat shock protein 90 (a chaperone, implicated as master regulator of KIT in the GIST cell) inhibitors [28] and the histone deacetylase (HDAC) inhibitors that result in KIT oncoprotein destruction irrespective of the imatinib resistance status and flavopiridol [29] that blocks KIT gene transcription and thus impairs KIT synthesis. It is perceivable that the clinical efficacy of these novel therapeutic agents can also be objectively monitored by PET imaging with FDG and possibly other novel tracers.

Thus, with the potential of addressing multiple concerns effectively, the promise of PET-based molecular imaging in managing patients with GISTs will be increasingly realized that will be well beyond its proven role in monitoring therapeutic response. It is imperative that this will lead to further enhancement of molecular imaging-based GIST research and therapeutics that is presently at the forefront of the advances in the personalized medicine in the practice of oncology.

\section{References}

1. Basu S, Mohandas KM, Peshwe H, Asopa R, Vyawahare M. FDG-PET and PET/CT in the clinical management of gastrointestinal stromal tumor. Nucl Med Commun 2008;29(12):1026-39.

2. Demetri GD, von Mehren M, Blanke CD, Van den Abbeele AD, Eisenberg B, Roberts PJ, et al. Efficacy and safety of imatinib mesylate in advanced gastrointestinal stromal tumors. N Engl J Med 2002;347:472-80.

3. Stroobants S, Goeminne J, Seegers M, Dimitrijevic S, Dupont P, Nuyts J, et al. 18FDG-Positron emission tomography for the early prediction of response in advanced soft tissue sarcoma treated with imatinib mesylate (Glivec). Eur J Cancer 2003;39(14):201220.

4. Gayed I, Vu T, Iyer R, Johnson M, Macapinlac H, Swanston N, et al. The role of $18 \mathrm{~F}-\mathrm{FDG}$ PET in staging and early prediction of response to therapy of recurrent gastrointestinal stromal tumors. J Nucl Med 2004;45:17-21.

5. Antoch G, Kanja J, Bauer S, Kuehl H, Renzing-Koehler K, Schuette J, et al. Comparison of PET, CT, and dual-modality PET/ CT imaging for monitoring of imatinib (ST1571) therapy in patients with gastrointestinal stromal tumors. J Nucl Med 2004;45:357-65.

6. Jager PL, Gietema JA, van der Graaf WT. Imatinib mesylate for the treatment of gastrointestinal stromal tumours: best monitored with FDG PET. Nucl Med Commun 2004;25(5):433-8.

7. Basu S, Nair N, Peshwe H, Mohandas KM. Potential role of FDG-PET to assess response to imatinib mesylate therapy and detecting viable disease in gastrointestinal stromal tumors (GIST). J Nucl Med 2004;45(Suppl):89.

8. Choi H, Charnsangavej C, de Castro Faria S, Tamm EP, Benjamin RS, Johnson MM, et al. CT evaluation of the response of gastrointestinal stromal tumors after imatinib mesylate treatment: a quantitative analysis correlated with FDG PET findings. AJR Am J Roentgenol 2004;183(6):1619-28.

9. Goerres GW, Stupp R, Barghouth G, Hany TF, Pestalozzi B, Dizendorf E, et al. The value of PET, CT and in-line PET/CT in patients with gastrointestinal stromal tumours: long-term outcome of treatment with imatinib mesylate. Eur J Nucl Med Mol Imaging 2005;32(2):153-62.

10. Heinicke T, Wardelmann E, Sauerbruch T, Tschampa HJ, Glasmacher A, Palmedo H. Very early detection of response to imatinib mesylate therapy of gastrointestinal stromal tumours using 18fluoro-deoxyglucose-positron emission tomography. Anticancer Res 2005;25(6C):4591-4.

11. Goh BK, Chow PK, Chuah KL, Yap WM, Wong WK. Pathologic, radiologic and PET scan response of gastrointestinal stromal tumors after neoadjuvant treatment with imatinib mesylate. Eur J Surg Oncol 2006;32:961-3. Epub 2006 Jul 12.

12. Choi H, Charnsangavej C, Faria SC, Macapinlac HA, Burgess MA, Patel SR, et al. Correlation of computed tomography and positron emission tomography in patients with metastatic gastrointestinal stromal tumor treated at a single institution with imatinib mesylate: proposal of new computed tomography response criteria. J Clin Oncol 2007;25:1753-9.

13. Holdsworth $\mathrm{CH}$, Badawi RD, Manola JB, Kijewski MF, Israel DA, Demetri GD, et al. CT and PET: early prognostic indicators of response to imatinib mesylate in patients with gastrointestinal stromal tumor. AJR Am J Roentgenol 2007;189:W324-30. 
14. van Oosterom AT, Judson I, Verweij J, Stroobants S, Donato di Paola E, Dimitrijevic S, et al. Safety and efficacy of imatinib (STI571) in metastatic gastrointestinal stromal tumours: a phase I study. Lancet 2001;358(9291):1421-3.

15. Van Glabbeke MM, Owzar K, Rankin C, Simes J, Crowley J, GIST Meta-analysis Group (MetaGIST). Comparison of two doses of imatinib for the treatment of unresectable or metastatic gastrointestinal stromal tumors (GIST): a meta-analysis based on 1,640 patients (pts). Journal of Clinical Oncology, 2007 ASCO Annual Meeting Proceedings Part I. Vol 25, No. 18S (June 20 Supplement), 2007: 10004.

16. Chintalgattu V, Patel SS, Khakoo AY. Cardiovascular effects of tyrosine kinase inhibitors used for gastrointestinal stromal tumors. Hematol Oncol Clin North Am 2009;23(1):97-107.

17. Turrisi G, Montagnani F, Grotti S, Marinozzi C, Bolognese L, Fiorentini G. Congestive heart failure during imatinib mesylate treatment. Int J Cardiol 3 Aug 2009 [Epub ahead of print].

18. Miettinen M, Majidi M, Lasota J. Pathology and diagnostic criteria of gastrointestinal stromal tumors (GISTs): a review. Eur J Cancer 2002;38 Suppl 5:S39-51.

19. Corless CL, Fletcher JA, Heinrich MC. Biology of gastrointestinal stromal tumors. J Clin Oncol 2004;22(18):3813-25.

20. Meesters B, Pauwels PA, Pijnenburg AM, Vlasveld LT, van Driel Repelaer OJ. Metastasis in a benign duodenal stromal tumour. Eur J Surg Oncol 1998;24(4):334-5.

21. Kamiyama Y, Aihara R, Nakabayashi T, Mochiki E, Asao T, Kuwano $\mathrm{H}$, et al. 18F-fluorodeoxyglucose positron emission tomography: useful technique for predicting malignant potential of gastrointestinal stromal tumors. World J Surg 2005;29:1429-35.
22. Yamada M, Niwa Y, Matsuura T, Miyahara R, Ohashi A, Maeda $\mathrm{O}$, et al. Gastric GIST malignancy evaluated by $18 \mathrm{FDG}-\mathrm{PET}$ as compared with EUS-FNA and endoscopic biopsy. Scand J Gastroenterol 2007;42:633-41.

23. Van den Abbeele AD. The lessons of GIST_PET and PET/CT: a new paradigm for imaging. Oncologist 2008;13 Suppl 2:8-13.

24. Van den Abbeele AD, Badawi RE, Manola J, Morgan JA, Desai J, Kazanovicz A, et al. Effects of cessation of imatinib mesylate (IM) therapy in patients (pts) with IM-refractory gastrointestinal stromal tumors (GIST) as visualized by FDG-PET scanning. J Clin Oncol 2004;22(14):198.

25. Liegl B, Kepten I, Le C, Zhu M, Demetri GD, Heinrich MC, et al. Heterogeneity of kinase inhibitor resistance mechanisms in GIST. J Pathol 2008;216:64-74.

26. Sevinc A, Camci C, Yilmaz M, Buyukhatipoglu H. The diagnosis of C-kit negative GIST by PDGFRA staining: clinical, pathological, and nuclear medicine perspective. Onkologie 2007;30:645-8.

27. Basu S, Asopa R, Peshwe H, Mohandas KM. Diffuse intense FDG uptake in the bone marrow in gastrointestinal stromal tumor with coexistent polycythemia rubra vera. Clin Nucl Med 2008;33 (11):782-3.

28. Bauer S, Yu LK, Demetri GD, Fletcher JA. Heat shock protein 90 inhibition in imatinib-resistant gastrointestinal stromal tumor. Cancer Res 2006;66:9153-61.

29. Sambol EB, Ambrosini G, Geha RC, Kennealey PT, Decarolis P, O'Connor R, et al. Flavopiridol targets c-KIT transcription and induces apoptosis in gastrointestinal stromal tumor cells. Cancer Res 2006;66:5858-66. 